\title{
Aquatic ecosystems of the lower reaches of the Zarafshan River. Diversity and ecological groups of molluscs
}

\author{
Khusniddin Baymuradov ${ }^{1, *}$, Tozagul Zhabborova ${ }^{2}$, Iroda $_{\text {Tuinazarova }}^{3}$, Bekzod Otakulov ${ }^{1}$, \\ Azamat Egamkulov ${ }^{1}$ \\ ${ }^{1}$ Samarkand State University \\ ${ }^{2}$ Karshi State University \\ ${ }^{3}$ Jizzakh Polytechnic Institute
}

\begin{abstract}
Aquatic ecosystems of the lower reaches of the Zarafshan River revealed the distribution of 49 species and 2 subspecies of molluscs. They are distributed over aquatic ecosystems as follows: in the lower reaches of the Zarafshan River, 32 species and 2 subspecies are distributed, in the Navbakhorsky fish farm - 22 species and in the Khatyrchinsky fish farm 15 species, in the Nurbuloksaysky fish farm - 22 species and in the Maidonsoysky - 18 species of mollusks. Their belonging to pelolymnophilic, peloreophilic, rheophilic, crenophilic, phytophilic, madicol, limno-crenophilic and telmatophilic ecological groups was considered.
\end{abstract}

\section{Topic relevance}

The study of the fauna of aquatic ecosystems in the lower reaches of the Zarafshan River creates important prerequisites for a comprehensive solution of theoretical and practical problems $[1,2,4]$. The diversity of the environment in aquatic ecosystems has created conditions for the distribution of many rare, endemic and relic animals $[3,5,6]$. At present, one of the urgent problems is the study of the diversity of mollusks and ecological groups of aquatic ecosystems in the lower reaches of the Zarafshan River.

\section{Research object and methods}

Below is a comprehensive analysis of the distribution, ecological groups of mollusks, their habitat and the expansion of their ranges [7,8,9]. These mollusks were studied by V.I.Zhadin (1938, 1952), Ya.I. Starobogatov, Z.I. Izatullaev (1984), Z.I. Izatullaev, Kh.T. Baimurodov (2009) and we used the system developed by Z. Izatullaev for Central Asian molluscs.

\section{Research results}

\footnotetext{
* Corresponding author: boymurodov1971@mail.ru
} 
Aquatic ecosystems in the lower reaches of the Zarafshan River, hydrographic conditions are complex. There are such sources of water as large and small rivers, lakes, springs and reservoirs, ponds. They differ from each other in a variety of hydrological, physicochemical and hydrobiological regimes. Each type of water is inhabited by molluscs, and each of them has its own ecological groups. It was found that 51 species and 2 subspecies of mollusks inhabit the aquatic ecosystems of the lower Amu Darya (Table 1).

Table 1. Aquatic ecosystems of the lower reaches of the Zarafshan River. Diversity and ecological groups of molluscs

\begin{tabular}{|c|c|c|c|c|c|c|c|}
\hline $\begin{array}{l}\text { No } \\
\text { • }\end{array}$ & Family and species & 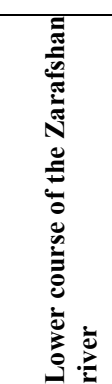 & 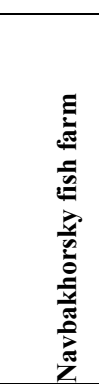 & 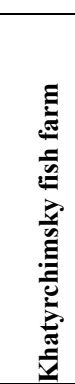 & 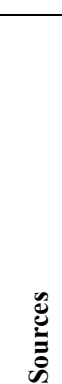 & 品 & Ecological groups \\
\hline & $\begin{array}{l}\text { Bivalvia class } \\
\text { Unionidae family } \\
\text { Sinanodonta genus }\end{array}$ & & & & & & \\
\hline 1 & Sinanodonta gibba & + & + & - & - & - & Peloreophile \\
\hline 2 & Sinanodonta orbicularis & + & - & + & - & - & Peloreophile \\
\hline 3 & Sinanodonta puerorum & + & - & + & - & - & Peloreophile \\
\hline & Colletopterum genus & & & & & & \\
\hline 4 & Colletopterum bactrianum & + & - & + & - & - & Rheophile \\
\hline 5 & Colletopterum cyreum sogdianum & + & - & + & - & - & Rheophile \\
\hline 6 & $\begin{array}{ll}\begin{array}{l}\text { Colletopterum } \\
\text { volgense }\end{array} & \text { ponderosum } \\
\end{array}$ & + & - & + & - & - & Pelolimnophile \\
\hline & \multicolumn{7}{|l|}{ Corbiculidae family Corbicula genus } \\
\hline 7 & Corbicula cor & + & + & + & - & - & Peloreophile \\
\hline 8 & Corbicula fluminalis & + & + & - & - & - & Peloreophile \\
\hline \multirow[t]{2}{*}{9} & Corbicula purpurea & + & - & + & - & - & Peloreophile \\
\hline & Corbiculina sort & & & & & & \\
\hline 10 & Corbiculina tibetensis & + & - & + & - & - & Peloreophile \\
\hline \multirow[t]{2}{*}{11} & Corbiculina ferghanensis & - & + & + & - & - & Peloreophile \\
\hline & \multicolumn{7}{|l|}{ Sphaeridae family Musculium genus } \\
\hline 12 & M.hungarica & + & - & - & + & - & Pelolimnophile \\
\hline & \multicolumn{7}{|l|}{ Euglesidae family Euglesa genus } \\
\hline 13 & Euglesa hissarica & - & - & - & + & - & Pelolimnophile \\
\hline 14 & Euglesa heldreichi & - & - & - & - & + & Peloreophile \\
\hline 15 & Euglesa turkestanica & - & - & - & + & - & Pelolimnophile \\
\hline 16 & Euglesa obliquata & - & - & - & - & + & Pelolimnophile \\
\hline \multirow[t]{2}{*}{17} & Euglesa turanica & - & - & - & - & + & Pelolimnophile \\
\hline & \multicolumn{7}{|c|}{ Pisidiidae family Odhneripisidium genus } \\
\hline 18 & Odhneripisidium behningi & - & - & - & + & + & Crenophile \\
\hline & Kuiperipisidium genus & & & & & & \\
\hline 19 & Kuiperipisidium terekense & - & - & - & + & + & Crenophile \\
\hline 20 & Kuiperipisidium issykkulense & - & - & - & + & - & Crenophile \\
\hline
\end{tabular}




\section{ITEEA 2021}

\begin{tabular}{|c|c|c|c|c|c|c|c|}
\hline 21 & Kuiperipisidium sogdianum & - & - & - & + & - & Crenophile \\
\hline \multirow[t]{2}{*}{22} & Kuiperipisidium polytimeticum & - & - & - & + & - & Crenophile \\
\hline & \multicolumn{7}{|c|}{ Gastopoda class Neritidae family Theodoxus genus } \\
\hline \multirow[t]{2}{*}{23} & Theodoxus.pallasi & + & - & - & + & - & Peloreophile \\
\hline & \multicolumn{7}{|c|}{ Valvatidae family Cincinna genus } \\
\hline 24 & Cincinna.pamirensis & + & - & - & - & - & Pelolimnophile \\
\hline \multirow[t]{2}{*}{25} & Cincinna.piscinalis & + & - & - & - & - & Pelolimnophile \\
\hline & \multicolumn{7}{|c|}{ Belgrandellidae family Bucharamnicola genus } \\
\hline \multirow[t]{2}{*}{26} & Bucharamnicola.vucharica & + & + & - & + & - & Crenophile \\
\hline & \multicolumn{7}{|l|}{ Martensamnicola genus } \\
\hline 27 & Martensamnicola. Brevicula & & + & - & + & - & Crenophile \\
\hline \multirow[t]{2}{*}{28} & Martensamnicola.hissarica & + & + & - & + & - & Crenophile \\
\hline & \multicolumn{7}{|c|}{ Horatiidae family Sogdamnicola genus } \\
\hline 29 & Sogdamnicola. Pallida & & + & - & + & - & Crenophile \\
\hline \multirow[t]{2}{*}{30} & Sogdamnicola.shadini & + & - & - & + & - & Crenophile \\
\hline & \multicolumn{7}{|c|}{ Lymnaeidae family Lymnaea genus } \\
\hline 31 & Lymnaea. Stagnalis & + & + & - & - & - & Phytophile \\
\hline 32 & Lymnaea.impura & + & & - & - & - & Phytophile \\
\hline 33 & Lymnaea.oblonga & + & + & - & - & + & Madikol \\
\hline 34 & Lymnaea.goupili & & + & - & + & + & Madikol \\
\hline 35 & Lymnaea.thiesseae & + & + & - & - & + & Madikol \\
\hline 36 & Lymnaea.truncatula & + & + & + & + & + & Madikol \\
\hline 37 & Lymnaea.subangulata & & + & - & - & + & Madikol \\
\hline 38 & Lymnaea.almaatina & + & + & - & + & - & Limno-crenophile \\
\hline 39 & Lymnaea.bowelli & + & + & - & + & - & Limno-crenophile \\
\hline 40 & Lymnaea.tenera & + & + & + & - & + & Phytophile \\
\hline 41 & Lymnaea.rectilabrum & + & - & + & - & + & Phytophile \\
\hline 42 & Lymnaea.psilia & + & + & + & - & + & Phytophile \\
\hline 43 & Lymnaea.fontinalis & + & + & + & - & + & Phytophile \\
\hline \multirow[t]{2}{*}{44} & Lymnaea.lagotis & + & - & + & - & - & Telmatophile \\
\hline & \multicolumn{7}{|l|}{ Phisidae family Costatella genus } \\
\hline \multirow[t]{2}{*}{45} & Costatella. Acuta & + & + & - & + & - & Phytophile \\
\hline & \multicolumn{7}{|c|}{ Planorbidae family Planorbis genus } \\
\hline 46 & Planorbis. Planorbis & + & + & - & - & + & Phytophile \\
\hline \multirow[t]{2}{*}{47} & Planorbis.tangitarensis & + & - & - & - & + & Phytophile \\
\hline & \multicolumn{7}{|l|}{ Anisus genus } \\
\hline 48 & Anisus. Acronicus & - & + & - & + & + & Phytophile \\
\hline 49 & Anisus.albus & - & - & - & - & + & Phytophile \\
\hline 50 & Anisus.centralis & + & - & - & + & - & Pelolimnophile \\
\hline 51 & Anisus.albopersicus & + & - & - & + & - & Phytophile \\
\hline \multicolumn{2}{|c|}{ Total species } & 34 & 22 & 15 & 22 & 18 & \\
\hline
\end{tabular}

Note: + species present, - species not present 
In the middle of the XX century, the anthropogenic destruction of zoogeographic barriers on land caused the penetration of invasive species into the fauna of most territories, in particular, into the hydrofauna, which remained unchanged for a long time.

Aquatic ecosystems of the lower reaches of the Zarafshan River revealed the distribution of 49 species and 2 subspecies of molluscs. They are distributed over aquatic ecosystems as follows: in the lower reaches of the Zarafshan River, 32 species and 2 subspecies are distributed, in the Navbakhorsky fish farm - 22 species and in the Khatyrchinsky fish farm - 15 species, in the Nurbuloksaysky fish farm - 22 species and in the Maidonsoysky - 18 species of mollusks. Their belonging to pelolymnophilic, peloreophilic, rheophilic, crenophilic, phytophilic, madicol, limno-crenophilic and telmatophilic ecological groups was considered.

The shell size of the mollusk species does not directly affect their distribution over the river sections. Representatives of the family Unionidae, which includes large species, and the family Corbiculidae, which includes small species, mean that the accumulation of rivers in the middle reaches is important for the hydrological regime of rivers in the distribution of bivalve molluscs along river beds.

\section{References}

1. V.V. Bogatov, Abstacts of the cinference Mollusks of the Eastern Asia and Adjacents Seas. Vladivostok, 6-12 (2014)

2. V. V. Bogatov, Ya. I. Starobogatov, Ruthinica, 4(2), 147-150 (2004)

3. P. Bouchet, Inventorying the molluscan fauna of theworld: howfar togon, 180 (2007)

4. Kh. T. Baymurodov, Advanced studies in science: theory and practice, The Collection of Scholarly Papers Materials of the International Scientific Conference, 239-242 (2016)

5. Kh. T. Baymurodov, Journal of Environmental Science and Engineering, 2, 692-696 (2013)

6. Kh. T. Baymurodov, Oecologia Montana International journal, 10-12 (2013)

7. Kh. T. Baymurodov, Research publications: nature, ecology and national economy, 7(27), 43-52 (2015)

8. M. V. Chertoprud, Monitoring of stream pollution on Structure of macrobenthos, 17 (1999)

9. V. I. Yurishinets, Bull. of Zoology, 1, 79-84 (2001) 